\title{
Genetic diversity of a large set of horse breeds raised in France assessed by microsatellite polymorphism
}

\author{
Grégoire Leroy*1,2, Lucille Callède ${ }^{1,2}$, Etienne Verrier ${ }^{1,2}$, Jean- \\ Claude Mériaux ${ }^{3}$, Anne Ricard ${ }^{4}$, Coralie Danchin-Burge ${ }^{1,2}$ and \\ Xavier Rognon ${ }^{1,2}$
} \begin{abstract}
génétique des animaux, BP 52627, 31326 Castanet-Tolosan, France
Email: Grégoire Leroy* - gregoire.leroy@agroparistech.fr; Lucille Callède - lcallede@gmail.com; Etienne Verrier - etienne.verrier@agroparistech.fr; Jean-Claude Mériaux - Jean-Claude.Meriaux@jouy.inra.fr;

Anne Ricard - Anne.Ricard@toulouse.inra.fr; Coralie Danchin-Burge - coralie.danchin@inst-elevage.asso.fr;

Xavier Rognon - Xavier.Rognon@jouy.inra.fr

* Corresponding author
\end{abstract}

Address: ${ }^{1}$ AgroParisTech, UMR1236 Génétique et Diversité Animales, 16 rue Claude Bernard, F-75321 Paris, France, 2INRA, UMR1236 Génétique et Diversité Animales, 78352 Jouy-en-Josas, France, ${ }^{3}$ LABOGENA, F-78352 Jouy-en-Josas, France and ${ }^{4}$ INRA, UR631 Station d'amélioration

Published: 19 March 2009

Genetics Selection Evolution 2009, 41:31 doi:|0.1186/I297-9686-4I-31

This article is available from: http://www.gsejournal.org/content/4I/I/3I

(c) 2009 Leroy et al; licensee BioMed Central Ltd.

This is an Open Access article distributed under the terms of the Creative Commons Attribution License (http://creativecommons.org/licenses/by/2.0), which permits unrestricted use, distribution, and reproduction in any medium, provided the original work is properly cited.
Received: 12 March 2009

Accepted: 19 March 2009

\begin{abstract}
After the recent publication of our article (Leroy, Genetics Selection Evolution 2009 4I:5), we found several errors in the published Table Three, concerning the computation of contribution to withinbreed diversity (CW). We apologize to the readers for these errors, which are corrected in the present erratum.
\end{abstract}

\section{Correction}

Table Three (see Table 1 of this erratum) of our recently published paper [1] contains several errors. Here we present the corrected version of Table Three (see Table 2 of this erratum) and explain the new data. The authors regret the errors.

\section{Results}

\section{Partition of diversity}

Errors concern the computation of the $C W$ component developed by Ollivier and Foulley [2]. In the new version, $C W$ ranged from -1 to 0.78 . As aggregate diversity $D$ is defined as a linear combination of $C W$ and contribution to between-breed diversity, column $D$ had also to be corrected, and ranged from -0.30 to 1.18 . Consequently, the Pearson correlation between $C W$ and $\Delta G D_{W S}$ was found to be -1 (instead of -0.72 in the previous version), and the 
Table I: Original and incorrect Table Three presented in Leroy et al. (2009)

\begin{tabular}{|c|c|c|c|c|c|c|c|c|c|c|c|}
\hline \multirow[t]{2}{*}{$\begin{array}{l}\text { Breed } \\
\text { code }\end{array}$} & \multicolumn{2}{|c|}{$\begin{array}{l}\text { Nb of breeding animals } \\
\text { in } 2005\end{array}$} & \multirow[t]{2}{*}{$\begin{array}{c}\mathrm{Pr} . \\
\text { extinction }\end{array}$} & \multicolumn{4}{|c|}{$\begin{array}{c}\text { Agregate diversity and cryopreservation } \\
\text { potential } \\
\text { (Ollivier and Foulley, 2005) }\end{array}$} & \multicolumn{4}{|c|}{$\begin{array}{l}\text { Loss or gain of diversity when a breed is } \\
\text { removed and contributions to optimal diversity } \\
\text { (Caballero and Toro, 2002) }\end{array}$} \\
\hline & Males & Females & & $C W$ & $C B$ & $D$ & $C P$ & $\Delta G D_{W S}$ & $\Delta G D_{B S}$ & $\Delta G D_{T}$ & $C_{i}$ \\
\hline $\mathrm{AA}$ & & & 0.11 & 0.35 & 0.85 & 0.39 & 0.10 & -0.0013 & -0.0018 & -0.0031 & $0 \%$ \\
\hline AR & 480 & 2130 & 0.03 & 0.29 & 10.90 & 1.25 & 0.35 & -0.0015 & -0.0010 & -0.0026 & $0 \%$ \\
\hline ARD & 187 & 1417 & 0.08 & -0.48 & 1.33 & -0.32 & 0.10 & 0.0031 & 0.0001 & 0.0032 & $0 \%$ \\
\hline AUX & 24 & 248 & 0.57 & -0.19 & 3.14 & 0.11 & 1.79 & 0.0023 & -0.0005 & 0.0018 & $0 \%$ \\
\hline BOUL & 58 & 540 & 0.24 & -0.27 & 12.35 & 0.87 & 2.95 & 0.0040 & -0.0023 & 0.0018 & $6 \%$ \\
\hline BR & 621 & 6380 & 0.02 & -0.38 & 5.57 & 0.16 & 0.12 & 0.0016 & 0.0009 & 0.0024 & $0 \%$ \\
\hline CAM & 118 & 837 & 0.12 & 0.00 & 7.99 & 0.73 & 0.97 & -0.0018 & 0.0013 & -0.0006 & $0 \%$ \\
\hline COBND & 63 & 760 & 0.21 & -0.06 & 2.42 & 0.16 & 0.52 & -0.0017 & 0.0019 & 0.0002 & $2 \%$ \\
\hline COMT & 856 & 7073 & 0.02 & -0.25 & 3.63 & 0.11 & 0.06 & 0.0000 & 0.0015 & 0.0015 & $0 \%$ \\
\hline LAND & 22 & 73 & 0.74 & 0.06 & 3.99 & 0.41 & 2.95 & -0.0029 & 0.0016 & -0.0014 & $2 \%$ \\
\hline MER & 93 & 1012 & 0.15 & -0.04 & 10.41 & 0.91 & 1.53 & 0.0000 & 0.0001 & 0.0001 & $0 \%$ \\
\hline PER & 183 & 2461 & 0.07 & -0.32 & 4.60 & 0.12 & 0.34 & 0.0006 & 0.0014 & 0.0020 & $0 \%$ \\
\hline PFS & 100 & 949 & 0.14 & 0.39 & 1.93 & 0.53 & 0.27 & -0.0055 & 0.0024 & -0.0031 & $70 \%$ \\
\hline POIT & 39 & 199 & 0.38 & -0.43 & 12.60 & 0.75 & 4.83 & 0.0069 & -0.0030 & 0.0039 & $0 \%$ \\
\hline POT & 94 & 910 & 0.15 & 0.19 & 1.33 & 0.29 & 0.20 & -0.0040 & 0.0024 & -0.0016 & $5 \%$ \\
\hline PS & 369 & 8049 & 0.04 & 0.50 & 6.17 & 1.02 & 0.22 & -0.0001 & -0.0041 & -0.0042 & $1 \%$ \\
\hline SF & 474 & 11700 & 0.03 & 0.45 & 1.33 & 0.53 & 0.04 & -0.0024 & -0.0013 & -0.0037 & $15 \%$ \\
\hline TDN & 16 & 183 & 0.85 & -0.17 & 1.93 & 0.02 & 1.64 & 0.0032 & -0.0009 & 0.0022 & $0 \%$ \\
\hline TF & 527 & 15950 & 0.02 & 0.36 & 7.51 & 1.01 & 0.18 & -0.0002 & -0.0029 & -0.0032 & $0 \%$ \\
\hline Sum & & & & 0 & 100 & 9.054 & & 0 & -0.043 & 0.043 & $100 \%$ \\
\hline
\end{tabular}

$C W=$ contribution to within-breed diversity; $C B=$ contribution to between-breed diversity; $D=$ aggregate diversity; $C P=$ cryopreservation potential; $\Delta G D_{W S}=$ loss or gain of gene diversity within populations when breed is removed; $\Delta G D_{B S}=$ loss or gain of gene diversity between populations when breed is removed; $\Delta G D_{T}=$ loss or gain of total diversity when the breed is removed; $C_{i}=$ contribution of the breed to optimise $G D_{T}$

Table 2: Corrected Table Three

\begin{tabular}{|c|c|c|c|c|c|c|c|c|c|c|c|}
\hline \multirow[t]{2}{*}{$\begin{array}{l}\text { Breed } \\
\text { code }\end{array}$} & \multicolumn{2}{|c|}{$\begin{array}{l}\text { Nb of breeding animals } \\
\text { in } 2005\end{array}$} & \multirow[t]{2}{*}{$\begin{array}{c}\text { Pr. } \\
\text { Extinction }\end{array}$} & \multicolumn{4}{|c|}{$\begin{array}{c}\text { Agregate diversity and cryopreservation } \\
\text { potential } \\
\text { (Ollivier and Foulley, 2005) }\end{array}$} & \multicolumn{4}{|c|}{$\begin{array}{l}\text { Loss or gain of diversity when a breed is } \\
\text { removed and contributions to optimal diversity } \\
\text { (Caballero and Toro, 2002) }\end{array}$} \\
\hline & Males & Females & & $C W$ & $C B$ & $D$ & $C P$ & $\Delta G D_{W S}$ & $\Delta G D_{B S}$ & $\Delta G D_{T}$ & $C_{i}$ \\
\hline $\mathrm{AA}$ & 119 & I 443 & 0.11 & 0.18 & 0.85 & 0.24 & 0.10 & -0.0013 & -0.0018 & -0.0031 & $0 \%$ \\
\hline AR & 480 & 2130 & 0.03 & 0.21 & 10.90 & 1.18 & 0.35 & -0.0015 & -0.0010 & -0.0026 & $0 \%$ \\
\hline ARD & 187 & 1417 & 0.08 & -0.46 & 1.33 & -0.30 & 0.10 & 0.0031 & 0.0001 & 0.0032 & $0 \%$ \\
\hline AUX & 24 & 248 & 0.57 & -0.32 & 3.14 & -0.01 & 1.79 & 0.0023 & -0.0005 & 0.0018 & $0 \%$ \\
\hline BOUL & 58 & 540 & 0.24 & -0.60 & 12.35 & 0.57 & 2.95 & 0.0040 & -0.0023 & 0.0018 & $6 \%$ \\
\hline BR & 621 & 6380 & 0.02 & -0.24 & 5.57 & 0.29 & 0.12 & 0.0016 & 0.0009 & 0.0024 & $0 \%$ \\
\hline CAM & 118 & 837 & 0.12 & 0.27 & 7.99 & 0.97 & 0.97 & -0.0018 & 0.0013 & -0.0006 & $0 \%$ \\
\hline COBND & 63 & 760 & 0.21 & 0.24 & 2.42 & 0.44 & 0.52 & -0.0017 & 0.0019 & 0.0002 & $2 \%$ \\
\hline COMT & 856 & 7073 & 0.02 & -0.01 & 3.63 & 0.32 & 0.06 & 0.0000 & 0.0015 & 0.0015 & $0 \%$ \\
\hline LAND & 22 & 73 & 0.74 & 0.48 & 3.99 & 0.79 & 2.95 & -0.0029 & 0.0016 & -0.0014 & $2 \%$ \\
\hline MER & 93 & 1012 & 0.15 & 0.02 & 10.41 & 0.96 & 1.53 & 0.0000 & 0.0001 & 0.0001 & $0 \%$ \\
\hline PER & 183 & 2461 & 0.07 & -0.10 & 4.60 & 0.33 & 0.34 & 0.0006 & 0.0014 & 0.0020 & $0 \%$ \\
\hline PFS & 100 & 949 & 0.14 & 0.78 & 1.93 & 0.89 & 0.27 & -0.0055 & 0.0024 & -0.0031 & $70 \%$ \\
\hline POIT & 39 & 199 & 0.38 & -1.00 & 12.60 & 0.23 & 4.83 & 0.0069 & -0.0030 & 0.0039 & $0 \%$ \\
\hline РОT & 94 & 910 & 0.15 & 0.58 & 1.33 & 0.64 & 0.20 & -0.0040 & 0.0024 & -0.0016 & $5 \%$ \\
\hline PS & 369 & 8049 & 0.04 & 0.01 & 6.17 & 0.57 & 0.22 & -0.0001 & -0.0041 & -0.0042 & $1 \%$ \\
\hline SF & 474 & 11700 & 0.03 & 0.34 & 1.33 & 0.43 & 0.04 & -0.0024 & -0.0013 & -0.0037 & $15 \%$ \\
\hline TDN & 16 & 183 & 0.85 & $-0.4 I$ & 1.93 & -0.20 & 1.64 & 0.0032 & -0.0009 & 0.0022 & $0 \%$ \\
\hline TF & 527 & 15950 & 0.02 & 0.02 & 7.51 & 0.70 & 0.18 & -0.0002 & -0.0029 & -0.0032 & $0 \%$ \\
\hline Sum & & & & 0 & 100 & 9.054 & & 0 & -0.043 & 0.043 & $100 \%$ \\
\hline
\end{tabular}


aggregate diversity $D$, which are now MER, LAND and POT, instead of BOUL, MER and POIT.

Finally, since the discussion on breed conservation is based on the use of several other methods and parameters, the above new results do not change our recommendations on which breeds specifically need support.

\section{References}

I. Leroy G, Callede L, Verrier E, Mériaux JC, Ricard A, Danchin-Burge $C$, Rognon X: Genetic diversity of a large set of horse breeds raised in France assessed by microsatellite polymorphism. Genet Sel Evol 2009, 41:5.

2. Ollivier L, Foulley JL: Aggregate diversity: New approach combining within- and between-breed genetic diversity. Livest Prod Sci 2005, 95:247-254.

3. Caballero A, Toro MA: Analysis of genetic diversity for the management of conserved subdivided populations. Conserv Genet 2002, 3:289-299.

Publish with Biomed Central and every scientist can read your work free of charge

"BioMed Central will be the most significant development for disseminating the results of biomedical research in our lifetime. "

Sir Paul Nurse, Cancer Research UK

Your research papers will be:

- available free of charge to the entire biomedical community

- peer reviewed and published immediately upon acceptance

- cited in PubMed and archived on PubMed Central

- yours - you keep the copyright 